or spare time (sic) of researchers to mobilize data into the public domain.

One proposal discussed recently (http://tinyurl.com/ 6elpq4) concerned the building of a realistic measure for use of database elements and a 'cite me' button for a dynamic composite web page. The Global Biodiversity Information Facility (GBIF, www.gbif.org) is investigating the assignment of 'life-science identifiers' to allow accreditation not only to data sets, but also to the individual datum and its author. Simplified mechanisms are needed that make it easy for individuals to assign these identifiers to their data.

We believe that scientists' productivity also needs to be gauged through data publishing, which requires a culture change in the recognition of scientific output. An industry-standard identifier, such as that proposed by the GBIF, could be part of publishers' referencing systems, and authors could provide 'citation identifiers' for all data records and data sets. Such a mechanism would achieve increased data mobilization and increased accreditation, both desirable to scientists.

Dave Roberts The Natural History Museum, Cromwell Road, London SW7 5BD, UK

Vishwas Chavan GBIF Secretariat, Universitetsparken 15, DK-2100

Copenhagen, Denmark

\section{Name variations can hit citation rankings}

SIR - The Correspondence 'Give south Indian authors their true names' (Nature 452, 530; 2008) and earlier News Feature 'Identity crisis' (Nature 451, 766-767; 2008) are highly relevant to calculations of PubMed citations and $h$-index (the number $n$ of $a$ researcher's papers that have received at least $n$ citations).

For example, I used to use the south Indian form of my name:
T. Biji Kurien, with Biji being my personal name. I have seven publications cited incorrectly in PubMed as being by 'Kurien, T. B.', 'Bijikurien, T.' or 'Kurien, B.'. Four of these entries were cited often enough to be counted towards my h-index computation. As I had by then changed my name to conform with Western style, these publications unfortunately do not appear in the Web of Science or PubMed under my current name format. Consequently, my h-index ranking has fallen by $25 \%$.

It is of paramount importance to adhere to a consistent name pattern right from the start, in order to maintain a correct list of publications in the public databases as well as the right h-index rankings.

Biji T. Kurien Arthritis and Immunology, Oklahoma Medical Research Foundation, 825 NE 13th Street, Oklahoma City, Oklahoma 73104, USA

\section{Names: dropped to avoid prejudice, now useful again}

SIR - The Correspondence 'Give south Indian authors their true names' (Nature 452, 530; 2008), incorrectly states that people from the south do not traditionally have surnames.

I am from southern India and have a proper surname - as do all the families in my region. Besides Patil, surnames such as Naidu, Reddy, Rao and Gouda are common in the different states of southern India. One of the authors of the Correspondence has the surname Kutty.

Surnames have widely fallen into disuse because our fathers and forefathers avoided using them to prevent discrimination on grounds of caste.

It doesn't make sense in this case to use only an author's first name in scientific publications and to devise a special system to accommodate a different naming format. Instead, editors should encourage these authors to revive the use of their surnames.

Prabhu B. Patil E307, Centre for Cellular and Molecular Biology, Hyderabad 500007, India

Readers are welcome to comment at the Nature India blog Indigenus, http://tinyurl.com/58r9wf

\section{Open-access more harm than good in developing world}

SIR - The traditional 'publish for free and pay to read' business model adopted by publishers of academic journals can lead to disparity in access to scholarly literature, exacerbated by rising journal costs and shrinking library budgets. However, although the 'pay to publish and read for free' business model of open-access publishing has helped to create a level playing field for readers, it does more harm than good in the developing world.

Authors by no means have a level playing field, even in the traditional publishing model. The dynamics of peer review make it hard to ensure that publication of an article is a function of only its quality, uninfluenced by factors such as topicality or the author's name and affiliation. The openaccess model makes the playing field for authors even more uneven.

Page charges may be waived for authors who cannot afford to pay, but a model that depends on payment by authors can afford only a few such waivers. And why should anyone want to survive on charity? The argument that it is the granting agency and not the author that pays does not wash either. If anything, the playing field for grants is even more uneven. Besides, this will undermine, rather than encourage, the whole area of grant-free research.

Page charges make extra difficulties for authors, while the old problems associated with peer review persist. They could be disastrous for the underdeveloped world, encouraging people to remain as consumers (readers), rather than to become producers (authors) of knowledge.

A 'publish for free, read for free' model may one day prove to be viable. Meanwhile, if I have to choose between the two evils, I prefer the 'publish for free and pay to read' model over the 'pay to publish and read for free' one. Because if I must choose between publishing or reading, I would choose to publish. Who would not? Raghavendra Gadagkar Centre for Ecological Sciences, Indian Institute of Science, Bangalore 560012, India

\section{$A 3 D$ revolution in communicating science}

SIR - Since the release of Adobe Systems' Portable Document Format (PDF) version 1.6 in 2004 , it has become possible to view interactively three-dimensional models that are embedded into PDF files. This attribute will dramatically increase information content as well as data transparency in scientific papers. Additionally, replacing multiple two-dimensional figures of a three-dimensional structure with one integrated interactive threedimensional model will reduce the need for supplementary material.

The potential of this technological advance for all science is obvious. Because of the foreseeable rise in demand by the scientific community, publishers and scientific institutions need to work hand in hand to support the implementation of this highly desirable technique.

Jérôme Murienne UMR 5202,

Département Systématique et Evolution, case 50, Muséum national d'Histoire naturelle, 45 rue Buffon, 75005 Paris, France

Alexander Ziegler Institut für Biologie, Freie Universität Berlin, Königin-LuiseStraße 1-3, 14195 Berlin, Germany Bernhard Ruthensteiner Zoologische Staatssammlung München, Münchhausenstraße 21, 81247 München, Germany 